\title{
FT-IR, Thermal and NLO Studies on Amino Acid (L-Arginine and L-Alanine) Doped KDP Crystals
}

\author{
G.G. Muley ${ }^{a, *}$, M.N. RodE ${ }^{b}$ And B.H. PAWAR ${ }^{a}$ \\ ${ }^{a}$ Department of Physics, Sant Gadge Baba Amravati University, Amravati-444602 (MS), India \\ ${ }^{b}$ Vaidhyanath College Parli-V, Dist. Beed (MS), India
}

(Received July 24, 2009; in final form October 12, 2009)

\begin{abstract}
Single crystals of pure and amino acid (L-arginine and L-alanine) doped potassium dihydrogen phosphate (KDP) for second harmonic generation have been grown from low temperature solution growth method by employing slow evaporation of the solvent. FT-IR spectrum of pure and doped KDP crystals confirms qualitatively the doping of the L-arginine and L-alanine in the host crystals. UV-visible spectroscopy shows the improvement in the transparency. Crystal structure has been studied by powder X-ray diffraction. Modification in the lattice parameters has been observed. Improvement in the SHG efficiency was studied by the Kurtz and Perry method. Thermal analysis has been performed on the grown crystals.
\end{abstract}

PACS numbers: $61.66 . \mathrm{Hq}$

\section{Introduction}

The numerous applications of the nonlinear optical (NLO) crystals in the vast field of science and technology [1-6] made the process of search of the new NLO crystals and improvements in the properties of the known crystals a never stopping process. KDP crystal is widely used and thoroughly studied NLO crystal. Many researchers have tried to modify the properties and growth rate of the KDP crystal by either changing the growth conditions or by adding different impurities. The NLO and other properties of the crystal have been improved by doping of organic impurities [7-34]. The KDP crystal has been grown with high growth rate as much as $50 \mathrm{~cm}$ per day [35].

Kumaresan et al. [36] have grown the amino acids such as L-glutamic acid, L-histidine and L-valine doped KDP crystals. They found improved NLO properties of the KDP crystal, modifications in the structure, optical, mechanical, and electrical properties, too. Parikh et al. [37] and Kumaresan et al. [38] have studied L-arginine doped KDP crystal. They have investigated the effect of the doping on the NLO, thermal, mechanical properties and optical transparencies. In the present work, the dopant level of L-arginine in KDP has been optimized for the first time and it is found to be $4 \mathrm{~mol} \%$. No single reference has been observed for attempting the doping of L-alanine in KDP. In the present work, the growth of the L-arginine and L-alanine doped KDP crystals, its characterization and findings have been reported.

* corresponding author; e-mail: gajananggm@yahoo.co.in

\section{Experimental}

\subsection{Solubility study}

It is desirable to study the solubility of the material in a suitable solvent before proceeding for the crystal growth. Solubility must be moderate and should have positive temperature gradient in a selected solvent. Solubility of the pure and doped KDP in water was studied gravimetrically. The solutions of pure and doped KDP were prepared separately and kept in a constant temperature bath for two hours with constant stirring. $50 \mathrm{ml}$ solution of each sample was taken and dried in oven to measure the dissolved solute. Same process was repeated for different temperature. The slight change in the solubility has been observed for doped KDP as compared to the pure KDP in water. Further, the solubility has positive gradient with temperature. Thus the double distilled water was used as a solvent throughout the experiment. Solubility curves of the pure and L-arginine and L-alanine doped KDP crystals have been shown in Figs. 1 and 2 .

\subsection{Crystal growth}

The pure and doped KDP single crystals were grown by dissolving purified KDP, L-arginine and L-alanine powders in appropriate amount in double distilled water and heated at a constant temperature $43{ }^{\circ} \mathrm{C}$ with continuous stirring using magnetic stirrer for two hours to form a mother solution. The solution is then filtered using the Whatmann filter papers and kept in optically heated constant temperature bath of an accuracy $\pm 0.01^{\circ} \mathrm{C}$ at a temperature $40^{\circ} \mathrm{C}$ to harvest the seed crystals within $2-3$ days. The same process was used to prepare the solutions for the bulk crystal growth. Good quality seed crystals were used to grow the single crystals of bigger size within 


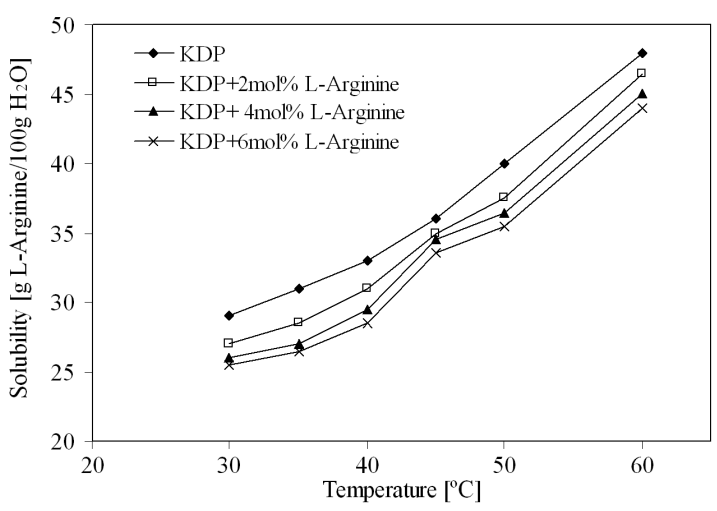

Fig. 1. Solubility curve of pure and L-arginine doped KDP.

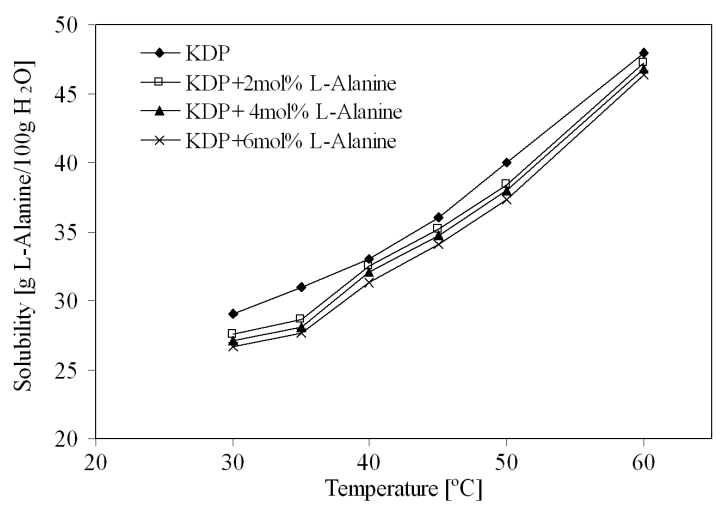

Fig. 2. Solubility curve of pure and L-alanine doped KDP.

10-15 days. In the experiment analytical reagent (AR) grade powders were used and further purified by repeated recrystallisation.

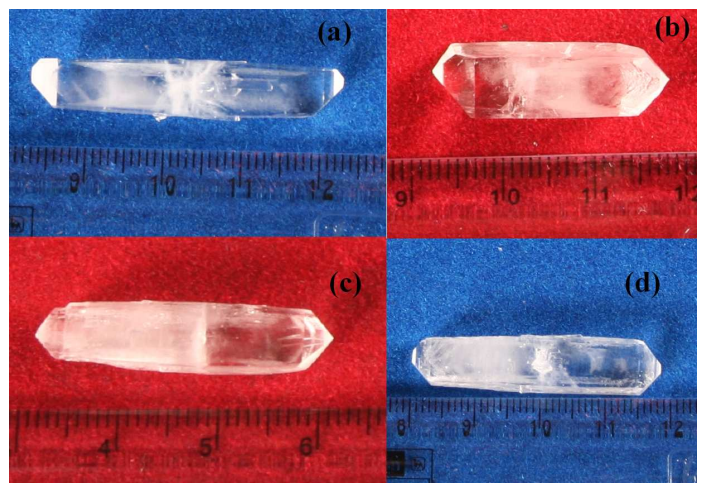

Fig. 3. Photographs of (a) $2 \mathrm{~mol} \%$ L-arginine,

(b) $6 \mathrm{~mol} \%$ L-arginine, (c) $4 \mathrm{~mol} \%$ L-alanine and

(d) $6 \mathrm{~mol} \%$ L-alanine doped KDP crystals.

Pure KDP crystal of size $38 \times 6 \times 5 \mathrm{~mm}^{3}$ having good optical quality was grown within 10 days by dissolving purified KDP in double distilled water. The $2 \mathrm{~mol} \%$,
$4 \mathrm{~mol} \%$ and $6 \mathrm{~mol} \%$ L-arginine doped KDP seed crystals and single crystals were independently grown by dissolving $2 \mathrm{~mol} \%, 4 \mathrm{~mol} \%$ and $6 \mathrm{~mol} \% \mathrm{~L}$-arginine and KDP powders in double distilled water separately. The $\mathrm{pH}$ of the solution was ranged from 4.2 to 4.4 . The single crystals of $2 \mathrm{~mol} \%, 4 \mathrm{~mol} \%$ and $6 \mathrm{~mol} \%$ L-arginine doped KDP of sizes $40 \times 6 \times 5 \mathrm{~mm}^{3}, 30 \times 7 \times 6 \mathrm{~mm}^{3}$ and $33 \times 7 \times 6 \mathrm{~mm}^{3}$ were grown within 10-15 days, respectively. The same process was used to grow the $2 \mathrm{~mol} \%$, $4 \mathrm{~mol} \%$ and $6 \mathrm{~mol} \%$ L-alanine doped KDP crystals of sizes $32 \times 10 \times 6 \mathrm{~mm}^{3}, 32 \times 7 \times 5 \mathrm{~mm}^{3}$ and $38 \times 9 \times 6 \mathrm{~mm}^{3}$, respectively. In this case, the $\mathrm{pH}$ of the solution was ranged from 4.3 to 4.5 . The photographs of some of the grown crystals have been shown in Fig. 3.

\subsection{Characterization}

The grown crystals were subjected to the Fourier transform infrared (FT-IR) study to verify the doping of the amino acid: L-arginine and L-alanine in KDP crystals. $\mathrm{X}$-ray diffraction (XRD) study was done to determine the crystal structures. Second harmonic generation (SHG) efficiency study was performed to measure the SHG efficiency of the grown crystals and thermal stability of the grown crystal was studied from thermal gravimetric analysis (TGA).

\section{Results and discussion}

\subsection{Morphology}

From the external observations of the pure and doped KDP crystals, it is clear that there is no change in the external morphology of the doped KDP crystals. During the crystal growth process continuous observations were taken in respect of growth rate over whole period of the growth. No detectable change in the growth rate of the doped crystals has been observed with reference to the pure KDP.

\subsection{SHG efficiency measurement}

The grown crystals were subjected to the NLO study to measure the SHG efficiency with respect to the pure KDP. To characterize the crystals, Kurtz and Perry method [39] was employed. In this experiment Q-switched, mode locked Nd:YAG laser of wavelength $1064 \mathrm{~nm}$ having pulse energy $2.35 \mathrm{~mJ}$, pulse duration $8 \mathrm{~ns}$ and repetition rate $10 \mathrm{~Hz}$ was used. The output was measured at $532 \mathrm{~nm}$ wavelength. The observed data has been tabulated in Table I.

The SHG efficiency found to be increased with the concentration of the L-arginine. The remarkable change in the SHG efficiency has been reported up to $4 \mathrm{~mol} \%$ doping of the L-arginine in KDP, which is in agreement with the results stated in Ref. [37]. For $6 \mathrm{~mol} \%$ doping remarkable increase in the SHG efficiency has not been observed. Also in the L-alanine doped KDP crystals, the SHG efficiency found to be increased with the concentration. The maximum SHG efficiency 1.67 has been reported for both 
TABLE I

SHG efficiencies of the pure, L-arginine and L-alanine doped KDP crystals.

\begin{tabular}{l|c|c}
\hline \hline & Measured output & SHG efficiency \\
\hline Pure KDP & $357 \mathrm{mV}$ & 1 \\
2 mol\% L-arginine doped KDP & $446 \mathrm{mV}$ & 1.25 \\
4 mol\% L-arginine doped KDP & $628 \mathrm{mV}$ & 1.76 \\
6 mol\% L-arginine doped KDP & $632 \mathrm{mV}$ & 1.77 \\
2 mol\% L-alanine doped KDP & $468 \mathrm{mV}$ & 1.31 \\
4 mol\% L-alanine doped KDP & $596 \mathrm{mV}$ & 1.67 \\
6 mol\% L-alanine doped KDP & $596 \mathrm{mV}$ & 1.67
\end{tabular}

$4 \mathrm{~mol} \%$ and $6 \mathrm{~mol} \%$ L-alanine doped KDP crystals. The increase in the SHG efficiencies is due to the weakening of the bond between $\mathrm{O}-\mathrm{H}$ and $\mathrm{C}=\mathrm{O}$ due to hydrogen bonding [36-38].

\subsection{Fourier transform infrared study}

The FT-IR spectrum of pure KDP, L-arginine and L-alanine doped KDP crystals (Fig. 4) have been recorded on Perkin Elmer FT-IR Spectrophotometer within the wavenumber range $600 \mathrm{~cm}^{-1}$ to $4000 \mathrm{~cm}^{-1}$. Pellets of the mixture of each sample with $\mathrm{KBr}$ have been prepared and used in the experiment.

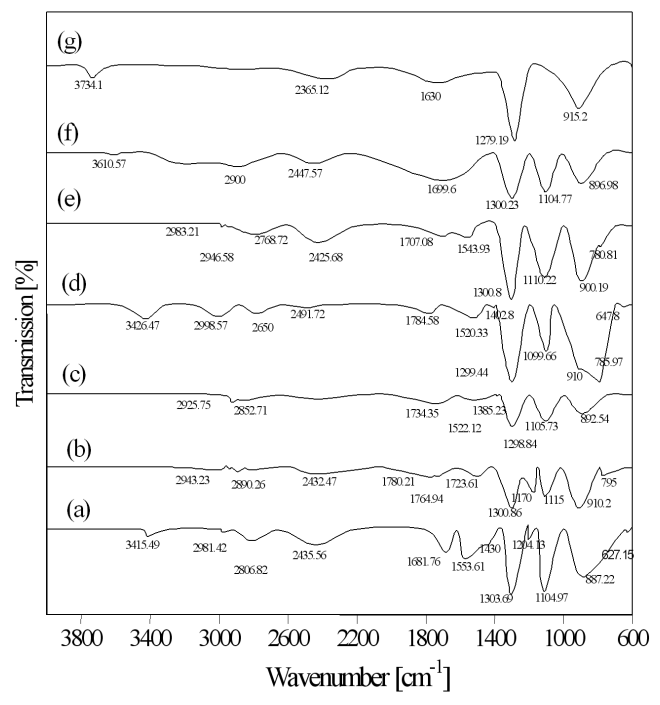

Fig. 4. FT-IR spectrum of (a) 2 mol\% L-alanine, (b) $4 \mathrm{~mol} \%$ L-alanine, (c) $6 \mathrm{~mol} \%$ L-alanine, (d) $2 \mathrm{~mol} \%$ L-arginine, (e) $4 \mathrm{~mol} \% \mathrm{~L}$-arginine, (f) $6 \mathrm{~mol} \% \mathrm{~L}$-arginine doped and $(\mathrm{g})$ pure KDP crystals.

TABLE II

Unit cell parameters.

\begin{tabular}{l|c|c|c}
\hline \hline & Crystal system & Space group & Unit cell parameters \\
\hline $\mathrm{KDP}$ & tetragonal & $I-42 d$ & $a=b=7.455 \AA, c=6.975 \AA, \alpha=\beta=\gamma=90^{\circ}$ \\
$6 \mathrm{~mol} \%$ L-arginine doped KDP & tetragonal & $I-42 d$ & $a=b=7.454 \AA, c=6.975 \AA, \alpha=\beta=\gamma=90^{\circ}$ \\
$4 \mathrm{~mol} \%$ L-alanine doped KDP & tetragonal & $I-42 d$ & $a=b=7.453 \AA, c=6.975 \AA, \alpha=\beta=\gamma=90^{\circ}$
\end{tabular}

In the FT-IR spectra of pure KDP crystal, the observed absorption peaks correspond to the $\mathrm{P}-\mathrm{OH}$ stretching, $\mathrm{P}-\mathrm{O}-\mathrm{H}$ bending, $\mathrm{P}=\mathrm{O}$ stretching, $\mathrm{P}-\mathrm{O}-\mathrm{H}$ stretching and $\mathrm{HO}-\mathrm{P}-\mathrm{OH}$ bending. In the FT-IR spectra of L-arginine and L-arginine doped KDP crystals, the same peaks have been observed with some additional peaks. These additional peaks correspond to the functional groups of L-arginine and L-alanine, which confirms the doping of the L-arginine and L-alanine in the KDP crystals (Table II). There is a slight shift in the peak positions because of the hydrogen bonding.

\subsection{Powder XRD study}

The powder XRD pattern (Fig. 5) of the pure and doped KDP crystals was recorded on X-ray diffractometer XPERT-PRO using $\mathrm{Cu} K_{\alpha}$ radiations (1.54060 $\AA$, $40 \mathrm{~mA}, 45 \mathrm{kV})$. The powder samples were scanned in steps of $0.0170^{\circ}$ for a time interval of 10.3359 s over a $2 \theta$ range of $10.0144-119.9874^{\circ}$. The data has been analyzed and unit cell parameters (Table III) have been calculated using software PowderX.

The XRD pattern of the $6 \mathrm{~mol} \% \mathrm{~L}$-arginine and $4 \mathrm{~mol} \%$ L-alanine doped KDP crystal confirms that the materials are crystalline and crystallize in the same crystal structure as that of pure KDP, but with very slight changes in the lattice parameters, which are in agreement with values cited in Ref. [37].

\subsection{UV-visible spectroscopy}

The UV-visible spectroscopy of the pure and doped KDP crystals was performed by using Shimadzu UV$1061 \mathrm{UV}$-visible spectrophotometer. Optically polished crystals of thickness $4 \mathrm{~mm}$ were used for this study. The transmissions and absorptions were measured over the wavelength range $190 \mathrm{~nm}$ to $900 \mathrm{~nm}$. The graphs for transmissions have been shown in Figs. 6 and 7 . 
TABLE III

FT-IR peak assignments [40, 41].

\begin{tabular}{c|c|c|c|l}
\hline \hline \multirow{2}{*}{$\begin{array}{c}\text { Pure KDP } \\
{\left[\mathrm{cm}^{-1}\right]}\end{array}$} & $2 \mathrm{~mol} \%$ & $4 \mathrm{~mol} \%$ & 6 mol\% & \multirow{2}{*}{ Assignments } \\
\cline { 2 - 4 } $3734.1,2365.12$ & $3426.47,2998.57$, & $2983.21,2946.58$, & $3610.57,2925.55$, & $\mathrm{P}-\mathrm{OH}$ stretching of $\mathrm{H}_{2} \mathrm{PO}{ }_{4}, \mathrm{O}-\mathrm{H}$ stretching of $\mathrm{COOH}$ \\
& $2777.71,2491.76$ & $2873.38,2768.72$, & $2852.71,2427.56$ & and water of crystallization, $\mathrm{N}-\mathrm{H}$ stretching of $\mathrm{NH} \mathrm{H}_{3}, \mathrm{C}-\mathrm{H}$ \\
& 1784.58 & 2425.68 & & stretching of $\mathrm{CH}_{2}$ and $\mathrm{CH}$ \\
1750.24 & 1299.44 & 1707.08 & 1699.60 & $\mathrm{C}=\mathrm{O}$ stretching, $\mathrm{P}-\mathrm{O}-\mathrm{H}$ bending and $-\mathrm{C}=\mathrm{NH} \mathrm{H}_{4}$ stretching \\
1279.19 & $1099.66,902.21$, & $1110.22,900.19$, & $1104.77,896.98$ & $\mathrm{CH}-\mathrm{OH}$ and $\mathrm{C}-\mathrm{H}$ stretching \\
915.20 & $877.70,785.97,647.80$ & 780.81 & & $\mathrm{P}-\mathrm{OH}$ deformation
\end{tabular}

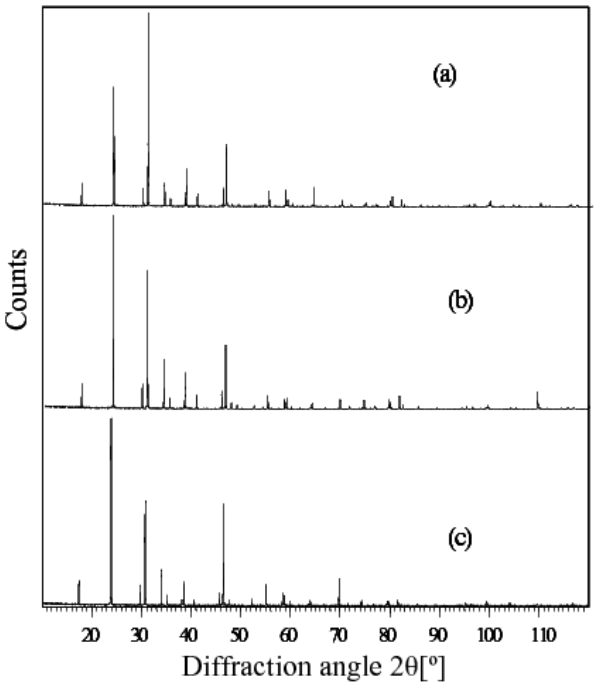

Fig. 5. Powder XRD Pattern of (a) pure, (b) $6 \mathrm{~mol} \%$ L-arginine and (c) $4 \mathrm{~mol} \%$ L-alanine doped KDP crystals.

The pure and L-arginine doped KDP crystals show a good transparency between $200 \mathrm{~nm}$ to $900 \mathrm{~nm}$. As expected, the transparency increases with the concentration of the L-arginine. For $4 \mathrm{~mol} \% \mathrm{~L}$-arginine doped KDP crystal, significant increase in transparency has been observed. For $6 \mathrm{~mol} \%$ L-arginine doped KDP crystal there is no considerable increase in the transparency with respect to $4 \mathrm{~mol} \%$ L-arginine doping. It is also found that the lower wavelength cut-off shifts somewhat toward longer wavelength. For pure KDP, the cut-off has been observed at $200 \mathrm{~nm}$ but for $4 \mathrm{~mol} \%$ and $6 \mathrm{~mol} \%$ doped KDP crystal it shows at $205 \mathrm{~nm}$.

For the L-alanine doped KDP crystals, the maximum transparency found to be for the $2 \mathrm{~mol} \%$ L-alanine doped KDP crystal. For $4 \mathrm{~mol} \%$ and $6 \mathrm{~mol} \%$ L-alanine doped KDP crystals the transparency is improved but not as much as that for $2 \mathrm{~mol} \%$ doped crystal. The effect of the doping on the lower wavelength cut-off has not been observed but doped crystals show sharp cut-off at $200 \mathrm{~nm}$ as compared to pure KDP.

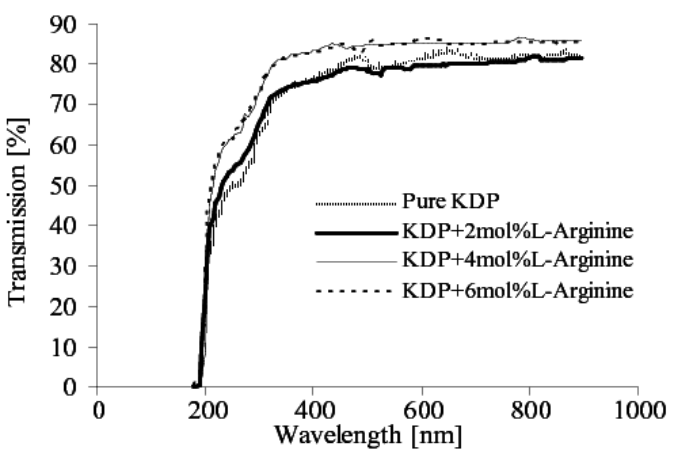

Fig. 6. UV-VIS-NIR spectra of pure and L-arginine doped KDP crystal.

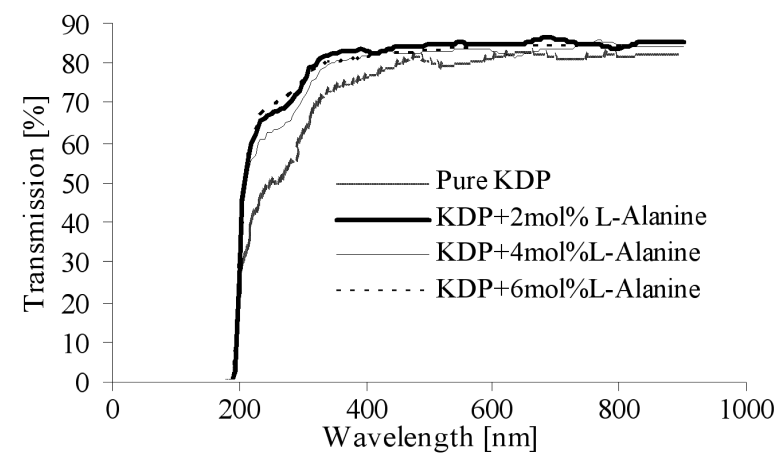

Fig. 7. UV-VIS-NIR spectra of pure and L-alanine doped KDP crystal.

\subsection{Thermal analysis}

The TGA curve of L-arginine and L-alanine doped and pure KDP crystals have been recorded on Perkin Elmer Dimmer TGDTA at a heating rate of $15^{\circ} \mathrm{C} / \mathrm{min}$ under argon atmosphere. For pure KDP crystal, after temperature about $230^{\circ} \mathrm{C}$ the weight loss starts due to the liberation of volatile substances, probably water molecule of decomposed KDP (Fig. 8) [37].

The TGA curve for $6 \mathrm{~mol} \% \mathrm{~L}$-arginine doped KDP crystal has been shown in Fig. 9. The experimental results show that the initial weight loss starts at about 


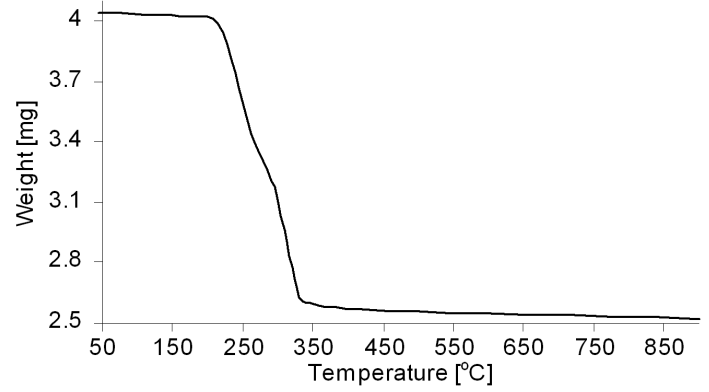

Fig. 8. TGA curve of pure KDP crystal.

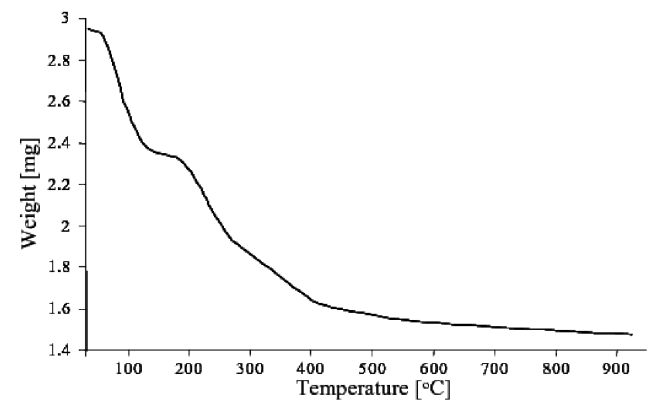

Fig. 9. TGA curve of 6 mol\% L-arginine doped KDP crystal.

$68^{\circ} \mathrm{C}$ and ends at $120^{\circ} \mathrm{C}$ with about $18 \%$ weight loss, which corresponds to the liberation of ammonia and water molecule. Second weight loss starts at about $204^{\circ} \mathrm{C}$. Rapid weight loss occurs up to $263^{\circ} \mathrm{C}$ and continues slowly up to $400^{\circ} \mathrm{C}$, which is possibly due to the decomposition of the KDP and remaining L-arginine.

The TGA curve of $4 \mathrm{~mol} \%$ L-alanine doped KDP crystal (Fig. 10) shows the maximum weight loss in the temperature range $205-365^{\circ} \mathrm{C}$. This is probably due to the decomposition of the KDP and L-alanine. Prolonged heating up to $900{ }^{\circ} \mathrm{C}$ does not produce any weight loss.

This study confirms the decrease in the thermal stability of KDP crystals with increasing doping level of L-arginine and L-alanine.

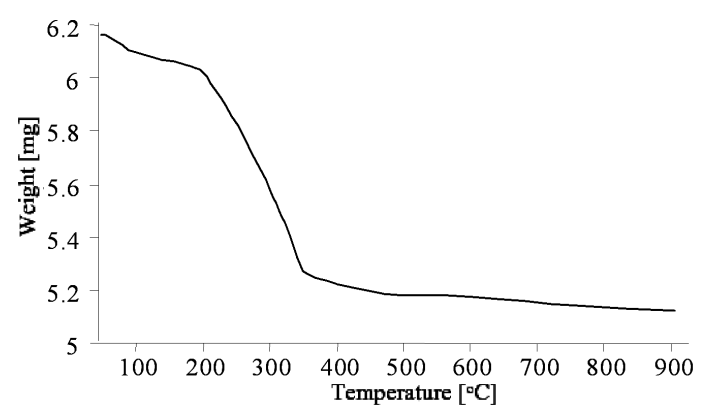

Fig. 10. TGA curve of $4 \mathrm{~mol} \%$ L-alanine doped KDP crystal.

\section{Conclusions}

Pure and amino acid (L-arginine and L-alanine) doped KDP crystals were grown from solution at a temperature $40^{\circ} \mathrm{C}$ by employing slow evaporation of the solvent. An external observation of the doped crystals shows no change in the morphology of the doped crystals and also no change in the growth rate as compared to the growth rate of the pure KDP crystal.

FT-IR Spectroscopy of the pure and doped KDP crystals confirms the expected doping of the L-arginine and L-alanine in the doped KDP crystals.

Pure and doped KDP crystals crystallizes in the same tetragonal crystal system and the lattice parameters calculated from the XRD pattern of the pure and doped KDP crystals show very slight changes.

The increasing doping level in the KDP crystal improves optical transparency. A remarkable increase in optical transparency has been observed up to $4 \mathrm{~mol} \%$ L-arginine doping. For $6 \mathrm{~mol} \%$ doping, no substantial change is observed in comparison with respect to $4 \mathrm{~mol} \%$ doping. In L-alanine doped crystals, the maximum transparency for $2 \mathrm{~mol} \%$ doped KDP crystal has been reported.

SHG efficiency test confirms the increase in the SHG efficiency in doped KDP crystals. For 6 mol\% L-arginine doped KDP crystal, the increase in the SHG efficiency with respect to $4 \mathrm{~mol} \%$ doping is found to be negligible. In L-alanine doped crystals, SHG efficiency has been found the same for both $4 \mathrm{~mol} \%$ and $6 \mathrm{~mol} \%$ doping.

TGA study confirms that the stability of the crystal decreases with the increasing concentration of the L-arginine and L-alanine in KDP crystals.

Considering the changes in the optical transparency, SHG efficiency and Thermal stability $4 \mathrm{~mol} \%$ L-arginine and $4 \mathrm{~mol} \%$ L-alanine doping in KDP crystal is found to be more suitable for optimum properties for SHG applications. $4 \mathrm{~mol} \%$ L-arginine and $4 \mathrm{~mol} \%$ L-alanine doped KDP crystal may be used for SHG purpose with improved SHG efficiency and optical transparency at the place of pure KDP crystals.

\section{References}

[1] H.M. Muncheryan, Lasers and Opto-electronic Devices, Hemisphere Pub. Co, New York 1991.

[2] Y.R. Shen, The Principles of the Nonlinear Optics, Wiley, New York 1984.

[3] P. Meystrey, M. Sargent II, Elements of Quantum Optics, Springer-Verlag, Berlin 1991.

[4] Jun-Ichi Sakai, Phase Conjugate Optics, McGraw Hill, Inc., New York, London 1992.

[5] P. Ramasamy, P. Santhana Raghavan, Crystal Growth Processes and Methods, KRU Publications, Kumbakonam 1999.

[6] P. Santhana Raghavan, P. Ramasamy, Recent Trends in Crystal Growth, Pinsa 68, New Delhi 2002, p. 235.

[7] P.F. Bordui, J.J. Zola, G. Kostecky, G.M. Loiacono, J. Cryst. Growth 71, 269 (1985). 
[8] S. Hirota, H. Miki, K. Fukui, K. Maed, J. Cryst. Growth 235, 541 (2002).

[9] L.M. Rouse, E.A.D. White, J. Cryst. Growth 34, 173 (1976).

[10] S. Veintemillas Verdaguer, R. Rodriguez Clemente, J. Cryst. Growth 79, 198 (1986).

[11] A.A. Zekic, M.M. Mitrovic, J. Cryst. Growth 258, 204 (2003).

[12] Xun Sun, Xinguang Xu, Zhangshou Gao, Youjun $\mathrm{Fu}$, Shenglai Wang, Hong Zeng, Yiping Li, J. Cryst. Growth 217, 404 (2000).

[13] Chen Jianzhong, Lin Sukun, Yang Fengtu, Wang Jiahe, Lang Jianming, J. Cryst. Growth 179, 226 (1997).

[14] S. Seif, K. Bhat, A.K. Batra, M.D. Aggarwal, R.B. Lal, Mater. Lett. 58, 991 (2004).

[15] I. Owczarek, K. Sangwal, J. Cryst. Growth 102, 574 (1990).

[16] O. Shimomura, J. Cryst. Growth 144, 253 (1994).

[17] A. Yokotani, H. Koide, T. Sasaki, T. Yamanaka, C. Yamanaka, J. Cryst. Growth 67, 627 (1984).

[18] Lu Taijing, R.B. Yalle, C.K. Ong, I. Sunagaw, J. Cryst. Growth 151, 342 (1995).

[19] P. Kumaresan, B.S. Moorthy Babu, P.M. Anbarasan, Mater. Res. Bull. 43, 1716 (2008).

[20] P. Triboulet, M. Cournil, J. Cryst. Growth 118, 231 (1992).

[21] S.K. Sharma, Sunil Verma, B.B. Shrivastava, V.K. Wadhawan, J. Cryst. Growth 244, 342 (2002).

[22] B. Dam, W.J.P. Van Enckevort, J. Cryst. Growth 69 306 (1984).

[23] K. Srinivasan, P. Ramasamy, A. Cantoni, G. Bocelli, Mater. Sci. Eng. B 52, 129 (1998).

[24] T.N. Thomas, T.A. Land, M. Johnson, W.H. Casey, J. Coll. Interface Sci. 280, 18 (2004).
[25] K. Srinivasan, S. Anbukumar, P. Ramasamy, J. Cryst. Growth 151, 226 (1995).

[26] N.P. Rajesh, V. Kannan, P. Santhana Raghavan, P. Ramasamy, C.W. Lan, Mater. Lett. 52, 326 (2002).

[27] K. Fujioka, S. Matsuo, T. Kanabe, H. Fujita, M. Nakatsuka, J. Cryst. Growth 181, 265 (1997).

[28] Guohui Li, Genbo Su, Xinxin Zhuang, Zhengdong Li, Youping He, J. Cryst. Growth 269, 443 (2004).

[29] Yang Shangfeng, Su Genbo, Tang Jing, Mao Bingwei, Wu Jianmin, Li Zhengdong, J. Cryst. Growth 203 , 425 (1999).

[30] R.J. Nelmes, G.M. Meyer, J.E. Tibballs, J. Phys. C, Solid State Phys. 15, 59 (1982).

[31] N. Zaitseva, L. Carman, I. Smolsky, R. Torres, M. Yan, J. Cryst. Growth 204, 512 (1999).

[32] A. Boukhris, C. Lecomte, B. Wyncke, F. Brehat, A. Thalal, J. Phys., Condens. Matter 6, 2475 (1994).

[33] P. Triboulet, M. Cournil, Mater. Chem. Phys. 24, 221 (1990).

[34] J. Podder, J. Cryst. Growth 237-239, 70 (2002).

[35] M. Nakatsuka, K. Fujioka, T. Kanabe, H. Fujita, J. Cryst. Growth 171, 531 (1997).

[36] P. Kumaresan, B.S. Moorthy Babu, P.M. Anbarasan, Opt. Mater. 30, 1361 (2008).

[37] K.D. Parikh, D.J. Dave, B.B. Parekh, M.J. Joshi, Bull Mater. Sci. 30, 105 (2007).

[38] P. Kumaresan, B.S. Moorthy Babu, P.M. Anbarasan, Opt. Adv. Mater. Rapid Commun. 1, 65 (2007).

[39] S.K. Kurtz, T.T. Perry, J. Appl. Phys. 39, 3798 (1968).

[40] W. Kemp, Organic Spectroscopy, Palgrave Foundation, New York, 2005.

[41] J.A. Dean, Lange's Handbook of Chemistry, 15th ed., McGraw-Hill, New York, 1998. 